\title{
Papulonecrotic tuberculids of the glans penis: case report
}

\author{
W JEYAKUMAR, * R GANESH,* M S MOHANRAM, $\dagger$ A SHANMUGASUNDARARAJ* \\ From the Departments of *Sexually Transmitted Diseases and †Dermatology, Government Rajaji Hospital, \\ Madurai, India
}

SUMMARY A case of papulonecrotic tuberculids on the glans penis alone is reported, and relevant reports published on this subject are reviewed.

Tuberculid results from an intense necrotising reaction caused by an extreme tissue response to isolated circulating Mycobacterium tuberculosis or its products in patients with a high level of immunity and sensitisation. The various types of tuberculids are: micropapular (lichen scrofulosorum), papular (papulonecrotic tuberculides), nodular (erythema induratum), and atypical nodular tuberculid.

Papulonecrotic tuberculids are common in young white people. They are chronic, recurrent, more or less symmetrically distributed, discrete, dusky red, inflammatory, papular eruptions leading to crust formation, ulceration, and scarring. They are neither painful nor pruritic. They occur in crops affecting the elbows, knees, legs, hands and feet, face and ears, buttocks, and penis. Patients with nodular tuberculid generally have erythrocyanotic circulation. Spontaneous resolution of the individual lesions occur with scarring. With effective antituberculous treatment, the crops of new lesions cease and the condition resolves.

The basic diagnostic criteria for papulonecrotic tuberculid are: a strongly positive Mantroux test result; chronic recurrent papular eruptions occurring in crops, with necrosis, ulceration, and scarring; a tuberculoid histology with endarteritis and thrombosis of the dermal vessels; and regression in response to antituberculous treatment.

\section{Case report}

A man aged 54, who had been married for 35 years, attended the sexually transmitted diseases department of this hospital in October 1985. During the previous 10 years he had had intermittent soreness of the penis.

\footnotetext{
Address for reprints: Dr W Jeyakumar, Department of Sexually Transmitted Diseases, Government Rajaji Hospital, Madurai 625020, Tamilnadu, India
}

Accepted for publication 23 July 1987
On examination the patient was moderately nourished and not anaemic. He was circumcised, and multiple soft, crusted, ulcers were present on the glans penis, coronal sulcus, and corona glandis. Multiple irregular pitted scars were noted on the same areas. There was no urethral discharge. The inguinal lymph nodes were enlarged, bilaterally discrete, and not tender. The scrotum and testes were normal. There were no other skin or mucous membrane lesions. He had had no extramarital sexual contact for 25 years, and gave no history of sexually transmitted disease (STD). He had no relevant constitutional symptoms and no history of such symptoms.

Serological tests for syphilis in October and November 1985 and February 1986 gave negative results. The white blood cell count was $9.0 \times 10^{9} / 1$, with a differential count of $72 \%$ polymorphs, $24 \%$ lymphocytes, and $4 \%$ eosinophils. The erythrocyte sedimentation rate was $6 \mathrm{~mm}$ in the first hour. The Mantoux test showed a positive result, with $20 \mathrm{~mm}$ induration. Radiological examination of the chest, renal tract, bones, and joints showed no abnormalities. A biopsy of the lesion on the glans penis showed dense chronic inflammatory cell filtrate with multiple tuberculoid granuloma formation consisting of epithelioid cells, lymphocytes and occasional giant cells, infiltration, and thickening of the vessel walls. The histology was consistent with papulonecrotic tuberculid. A lymph node biopsy showed reactive follicular hyperplasia and fibrosis.

The patient was treated with rifampicin $600 \mathrm{mg}$ daily, isoniazide $300 \mathrm{mg}$ daily, and ethambutol $800 \mathrm{mg}$ daily for six months and thereafter with isoniazid $300 \mathrm{mg}$ and ethambutol $800 \mathrm{mg}$ daily for one year. His spouse was screened for tuberculosis and for any other STD, but failed to show any positive findings. The lesions healed, and the Mantoux test was weakly reactive after six months. No recurrence was observed after March 1986. 


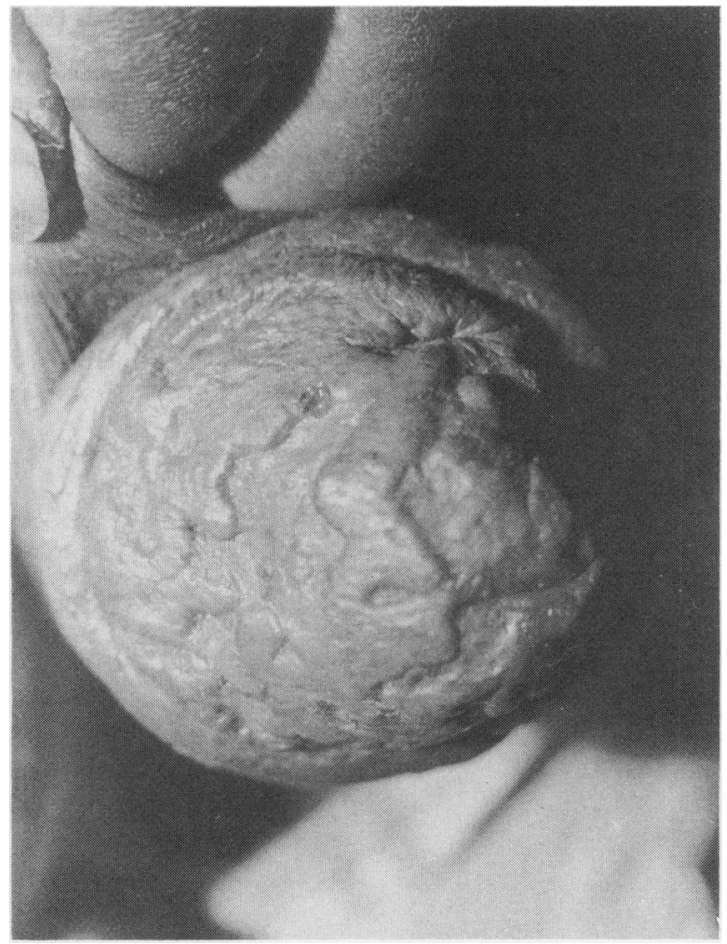

Fig 1 Glans penis with ulcers and pitted irregular scars before treatment.

\section{Discussion}

The exceptional occurrence of papulonecrotic tuberculid on the glans penis was first described by Hellerstrom and later by Bafverstedt and Hageman and Granroth. ${ }^{1}$ The common characteristic of tuberculid is the absence of tubercle bacilli, which may be due to their rapid destruction. The necrotic reaction of the papulonecrotic tuberculid may be due to chemicals liberated from different sources stimulating an immune response. It has been well established that dead tubercle bacilli or their fractions can evoke similar reactions to those produced by living organisms. ${ }^{2}$ The histological findings are said to show a small area of necrosis involving the upper dermis or the overlying epidermis. The area of necrosis is surrounded by an infiltrate largely non-specific in type but usually containing a tuberculoid structure at its periphery. Vascular changes are regularly present and consist of invasion of the walls of the vessels by an inflammatory infiltrate and thickening of the vessel walls. ${ }^{3}$ It is reasonable to expect genuine tuberculids to respond unequivocally to antituberculous measures. Papulonecrotic tuberculids belong to the hyperergic

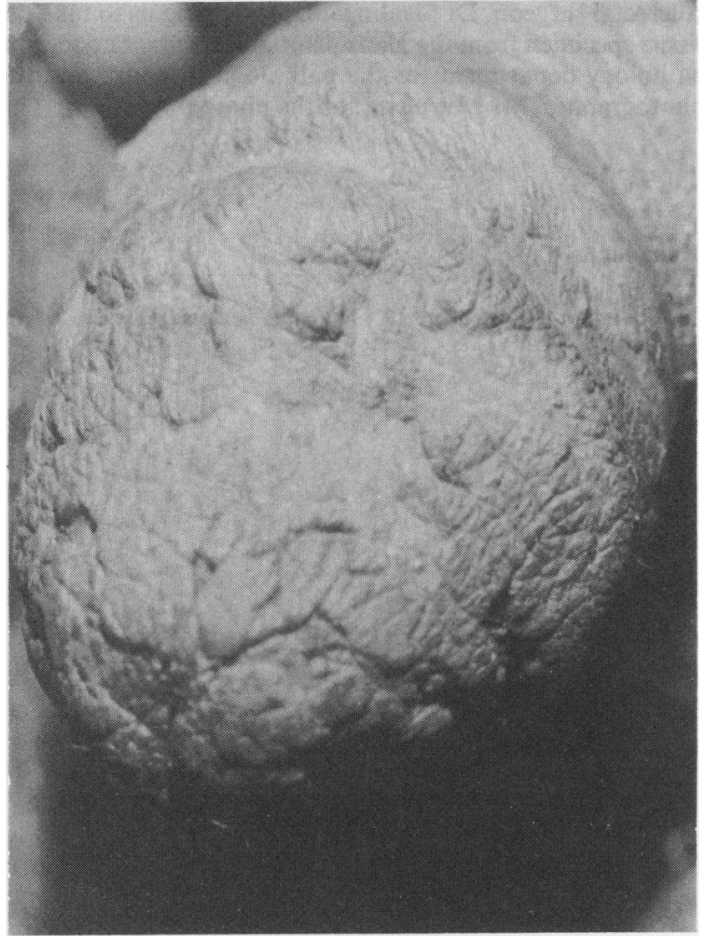

Fig 2 Glans penis with puckered scars after treatment.

group. The reaction usually occurs in the dermis. When the lesions are limited to the glans penis, the diagnosis can sometimes be difficult, and other conditions occurring on the glans penis, such as atypical soft sore, syphilis, recurrent herpes simplex, or cancer are differential diagnoses that should be considered. ${ }^{4}$

Wilson-Jones and Winkelmann cited a report of 91 cases of papulonecrotic tuberculid in 1976 in South Africa, where tuberculosis was still prevalent in the black population. ${ }^{5}$ Though tuberculids have become increasingly rare in countries where primary infections receive early and adequate treatment, follow up studies have shown that active tuberculosis was present nine years after the onset of tuberculids in $16 \%$ of 89 cases. Early dissemination and subsequent latency need not account for all cases. ${ }^{2}$ In India, where tuberculosis is still prevalent and subclinical tuberculosis is common, the existence of papulonecrotic tuberculid is possible, and awareness about this disease is particularly important when it affects only the genitals.

We thank the Dean of the Government Rajaji Hospital, Madurai, for permitting us to publish this case report, the 
132

colerectal surgeon, Dr Sivalingam, for helping us to take the tissue specimen from the glans penis and inguinal nodes, the pathology department for the pathological report, and the photographer, Mr Murugan, for the photographs.

\section{References}

1 Collomon PT, Wilson JP. The non venereal diseases of the
Jeyakumar, Ganesh, Mohanram, Shanmugasundararaj genitals. Springfield, Illinois, USA. Charles C Thomas Publisher, 1956:155.

2 Rook A, Wilkinson DS, Ebling FJG. Textbook of dermatology. Vol 1 2nd ed. Oxford: Blackwell Scientific Publication, 1972:624-5.

3 Lever WF, Lever GS. Histopathology of the skin. 5th ed. Philadelphia: JB Lippincott, 1975:282.

4 Stevanovic DV. Papulonecrotic tuberculids of the glans penis. Arch Dermatol 1958;78:760-1.

5 Wilson-Jones E, Winkelmann RK. Papulonecrotic tuberculid: a neglected disease in Western countries. J Am Acad Dermatol 1986;14:815-26. 